\title{
Enjeux et opportunités de la filière Silver économie : exemple de la France
}

\author{
Benjamin Zimmer
}

La Silver économie rassemble l'ensemble des acteurs producteurs de biens et/ou de services qui répondent aux besoins et aux usages des seniors et de leur entourage. C'est une filière transversale qui regroupe de nombreux secteurs d'activité allant de la santé en passant par la construction, les services, le cosmétique, la communication jusqu'au transport ou les loisirs, etc. et qui vise à créer de la valeur économique, sociale et sociétale. Cette approche économique du vieillissement présente de fortes opportunités de croissance, de production et d'emploi et est affichée comme une filière industrielle prioritaire par les pouvoirs publics.

\section{Herausforderungen und Chancen der "Silver Economy» - Beispiel Frankreich}

Die Silver Economy umfasst alle Warenhersteller und/oder Dienstleister, die die Bedürfnisse von Senioren und ihrem Umfeld erfüllen. Sie ist branchenübergreifend mit zahlreichen Tätigkeitsfeldern - von der Gesundheit über den Baubereich, Dienstleistungen, Kosmetik und Kommunikation bis hin zu Transport oder Freizeit usw. - und hat die Schaffung eines ökonomischen, sozialen und gesellschaftlichen Mehrwerts zum Ziel. Dieses auf Senioren bezogene wirtschaftliche Konzept verspricht große Chancen für Wachstum, Produktion und Beschäftigung und wird von öffentlichen Stellen als eine bevorzugte Industriebranche präsentiert.

DOI 10.1024/2297-5160/a000031

\section{Développement des écosystèmes propices à l'innovation}

Bien agir dans la Silver économie, adapter notre société au vieillissement, c'est également avoir une vision de l'innovation à $360^{\circ}$. Il nous faudra en effet innover sur le plan technologique (performances accrues et miniaturisation des accéléromètres d'un système de détection de chute), dans nos processus (coordination des professionnels de la santé et du médico-social), dans nos services (accessibilité du packaging des produits), dans l'usage des solutions (simplification des fonctionnalités des produits), dans nos organisations (nouveau canal de distribution, nouvelle organisation industrielle pour la production), dans notre modèle économique (processus d'achat public) et dans notre approche du vieillissement en tentant de donner aux seniors plus de vie à leurs années plutôt que plus d'années à leur vie. C'est à cette condition que l'ambition portée par ce projet de loi pourra s'exprimer. C'est à cette condition que le célèbre triple $\mathrm{A}$ « Accompagner, Anticiper et Adapter » de Michèle Delaunay se réalisera. Et, c'est en y répondant par un triple I « Innover, Investir et Industrialiser » que nous y arriverons.

Ainsi, de nombreuses entreprises ont saisi l'opportunité offerte par cette filière pour diversifier leurs solutions et conquérir ainsi de nouvelles parts de marché, d'autres se sont créées exclusivement pour cette filière (les pureplayers) pour définir de nouveaux marchés. Pour les accompagner dans leurs stratégies d'innovation des écosystèmes propices au développement de leurs innovations se sont structurés en France et dans le monde à l'image du cluster Silver Valley en Ile-de-France. Ces organisations innovantes ont pour principales fonctions d'être :

- Un accélérateur pour le développement et la mise sur le marché des innovations portées par des startups et PME;

- Un observatoire vivant des bonnes pratiques en termes d'innovations en développant une logique d'open innovation pour les ETI et grands groupes industriels ;

- Un moyen de valoriser les recherches des structures de recherches publiques et privées ;

- Un lieu permettant aux entreprises d'avoir accès aux investisseurs à tous les stades du développement : de 
l'amorçage au développement commercial à la transition industrielle ;

- Un outil qui favorise l'innovation tirée par les usages en faisant participer les bénéficiaires dès les phases amont de la conception de biens et services.

Ces écosystèmes se généralisent actuellement dans une conjoncture économique marquée par un ralentissement de la consommation des ménages. Effet collatéral de ce ralentissement, le mot ressource n'a jamais aussi bien porté son nom en contexte d'entreprise. Les ressources (financières, matérielles et humaines) sont ainsi plus limitées pour innover en contexte d'entreprise d'où l'apparition de ces structures.

\section{Vision stratégique de Silver Valley}

Silver Valley, association loi 1901 à but non lucratif, anime et fédère un écosystème de plus de 220 structures en Région Ile-de-France. Cette entité permet à de grandes entreprises d'accéder à différentes formes d'innovation conçues par de nombreuses startups et PME dans des secteurs d'activités diversifiés et réciproquement aux startups et PME d'accéder au savoir-faire et aux bonnes pratiques industrielles. Aussi, pour garantir leurs chances de succès sur des marchés donnés, Silver Valley permet à ces entreprises de coopérer avec des pôles de compétitivité, des syndicats, des grandes écoles ou universités, des collectivités, des utilisateurs, des fédérations, des cabinets de conseils ou des investisseurs.

L'innovation ouverte, incarnée par le modèle organisationnel de Silver Valley, vise à innover dans les produits, dans les services, dans les modèles économiques et dans de nouvelles organisations et processus (distribution, achat). C'est un moyen d'alimenter des stratégies pertinentes, de tester rapidement des solutions, d'identifier des bonnes pratiques particulièrement en amont de la conception de nouveaux biens et services, de sortir des sentiers battus, au moment où une filière industrielle se professionnalise.

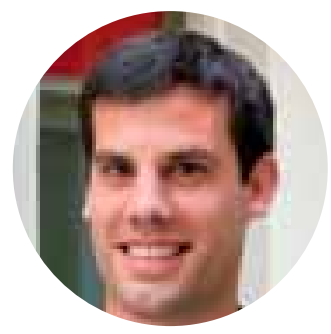

Benjamin Zimmer, Directeur de Silver Valley (www.silvervalley.fr). benjamin.zimmer@silvervalley.fr

\section{Universität} Zürich $^{\mathrm{UzH}}$

\section{Zentrum für Gerontologie}

\section{CAS Gerontologie heute besser verstehen, erfolgreich vermitteln, innovativ gestalten}

Das interdisziplinäre Zertifikatsprogramm vermittelt in 13 Tagesveranstaltungen das neueste gerontologische Grundlagenwissen in den Themenbereichen Alterstheorien, soziodemographische Entwicklung, psychische und somatisch verursachte Erkrankungen, Palliative Care, Gesundheitsförderung und Alterspolitik. Auch erweitert es die Methodenkompetenzen zur besseren Analyse und Vermittlung altersspezifischer Inhalte. Angesprochen sind Fachleute aus dem Bildungs-, Sozial- und Gesundheitsbereich, die sich auf die Planung und Vermittlung gerontologischer Fragestellungen und deren Besonderheiten spezialisieren möchten.

Dauer: 26. August 2016 bis 30. Januar 2017

Abschluss: Certificate of Advanced Studies in Gerontologie der Universität Zürich und 15 ECTS-Punkte

Information: Friederike Geray, Programmleitung,

Universität Zürich, Zentrum für Gerontologie, Pestalozzistrasse 24, 8032 Zürich,

www.zfg.uzh.ch (Weiterbildung), Tel. +41 4463534 24, friederike.geray@zfg.uzh.ch 